\title{
Characterisation of an RF Excited Argon Plasma Cathode Electron Beam Gun
}

\author{
Sofia del Pozo ${ }^{1,2,}$, Colin Ribton ${ }^{1}$, David Ryan Smith ${ }^{2}$ \\ ${ }^{1}$ Electron Beam Processes, TWI Ltd. \\ Granta Park, Great Abington, \\ Cambridge CB21 6AL, UK \\ ${ }^{2}$ Electronic \& Computer Engineering, \\ Brunel University, Uxbridge, \\ Middlesex UB8 3PH, UK \\ *Contact Author Email: eepgssd1@brunel.ac.uk
}

\begin{abstract}
This work describes the experimental set up used for carrying out spectroscopic measurements in a plasma cathode electron beam (EB) gun. Advantages of plasma cathode guns over thermionic guns are described. The factors affecting electron beam power such as plasma pressure, excitation power and plasma chamber geometry are discussed. The maximum beam current extracted was $53 \mathrm{~mA}$ from a $0.5 \mathrm{~mm}$ diameter aperture in the plasma chamber. In this work, the electron source is an argon plasma excited at $84 \mathrm{MHz}$. The pressure in the plasma chamber was measured to be within 0.9 to 1.2 mbar and was controlled by varying gas flow rates. The vacuum chamber was at a pressure of $10^{-5} \mathrm{mbar}$.
\end{abstract}

Keywords: electron sources; plasma devices; plasma diagnosis; emission spectroscopy; vacuum electronics.

\section{Introduction}

Advantages of the plasma cathode electron gun over conventional thermionic gun designs have been described and empirically demonstrated previously [1, 2]. Plasma cathode guns [3] avoid problems encountered with thermionic cathodes, mainly related to cathode erosion caused by ion bombardment coming from the work piece inside the vacuum chamber [4] and evaporation. Beam aberration caused by the use of a grid cup electrode to control the flow of electrons can be eliminated and by using pulse width modulation for beam power control a consistent beam profile can be maintained over the full power range. The plasma cathode is radio frequency excited, which allows switching the plasma and thus the electron beam very rapidly, i.e. 200 times faster than with conventional guns.

The plasma cathode electron gun device is described in [5]. The circuit consists of an RF inductively coupled circuit in which the RF signal is applied to a first antenna. The voltage generated in a second antenna circuit is applied to the plasma chamber. The gas fed into the chamber is ionized and the electrons in the plasma are extracted from the plasma chamber through a diaphragm by applying a high voltage gradient, e.g. $-60 \mathrm{kV}$. Figure 1 shows a picture of the plasma cathode gun body, which contains the plasma chamber and is assembled inside a vacuum chamber.

The EB power achieved with the plasma system is not as high as with conventional thermionic guns. The maximum power achieved to date has been $3 \mathrm{~kW}$. This is enough for some material processing applications such as surface modification and additive manufacturing of metallic parts. Higher beam powers are required for speeding up the additive manufacturing process or for welding of metals.

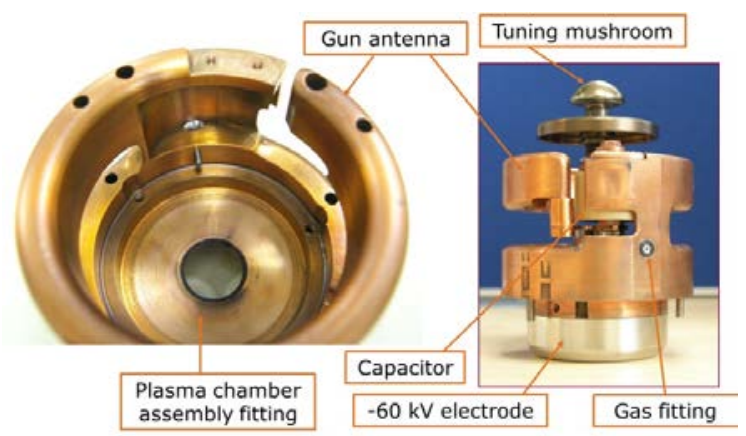

Figure 1. Plasma gun body showing top view (left) and front view (right). Reproduced by permission of TWI Ltd.

In order to investigate how to increase the beam power extracted from the plasma cathode electron gun, a more detailed study of the plasma and parameters affecting EB power is required. Optical emission spectroscopy is a passive method that allows real-time observation of the radiation emitted from atoms and molecules in the plasma [6]. This technique has been used to evaluate the plasma generated with argon gas, when operating at different plasma chamber pressures and excitation powers.

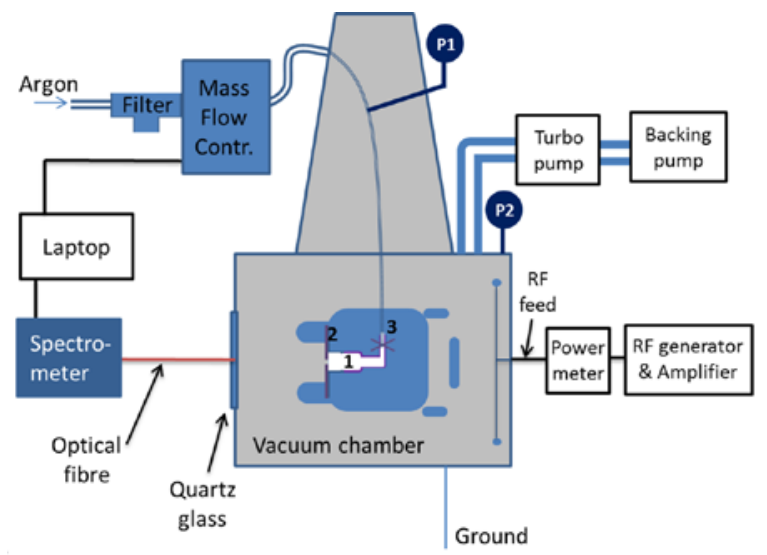

Figure 2. Schematic diagram showing the experimental setup for the plasma spectroscopic measurements. Reproduced by permission of TWI Ltd.

\section{Experimental Set Up and Preliminary Results}

Figure 2 shows the experimental setup used for investigating the plasma generated. 1 is the plasma chamber, 2 is the diaphragm, and 3 is a needle valve used to drop the pressure from the fore line to the plasma 


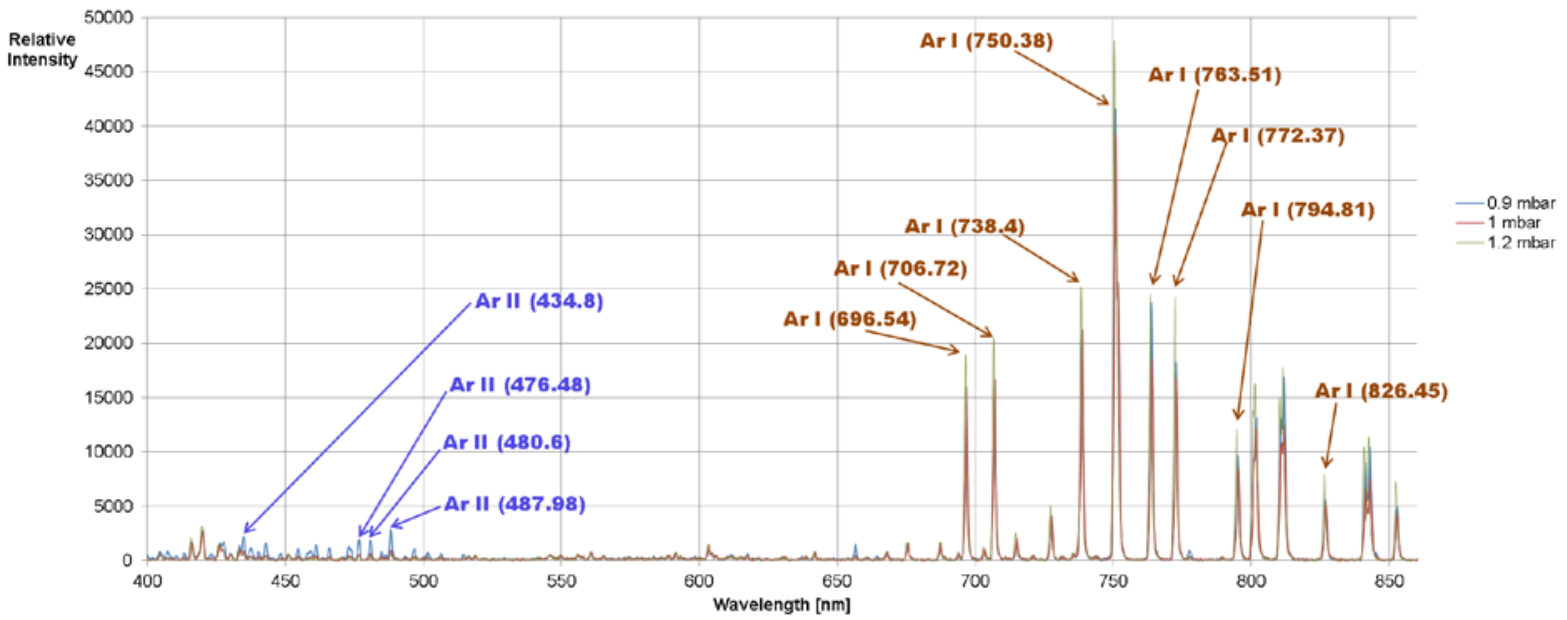

Figure 3. Spectroscopic measurements of the argon plasma generated in the plasma cathode EB gun at $25 \mathrm{~W}$ of forward power applied to the plasma chamber at three plasma chamber pressures: 0.9, 1 and 1.2 mbar. Reproduced by permission of TWI Ltd.

chamber. The following factors were expected to regulate regarding beam current extraction: electron density and sheath thickness of the plasma, strength of the electric field, gas pressure and plasma chamber geometry. The gas flow was accurately controlled by a mass flow controller and thus the stability of the plasma was highly improved. The pressure in the plasma chamber was measured at different flow rates. The equipment used for carrying out optical emission spectroscopy measurements comprised an Ocean Optics optical spectrometer USB4000 with enhanced sensitivity ( $1.5 \mathrm{~nm}$ FWHM optical resolution) and an optical fibre with a numerical aperture of $0.22 \pm 0.02$ (this yields an acceptance angle of $25.4^{\circ}$ in air) [7]. The equipment was used to record the emission radiation spectrum of the plasma across a wavelength range of 200 $\mathrm{nm}$ to $850 \mathrm{~nm}$. The emission spectra of the argon plasma cathode was recorded at plasma chamber pressures from 0.9 to 1.2 mbar and at $5 \mathrm{~W}$ to $50 \mathrm{~W}$ excitation powers. Figure 3 shows the emission spectra of the argon plasma cathode at 1.8, 5 and 10 sccms (estimated plasma pressures are $0.9,1$ and 1.2 mbar respectively) at $25 \mathrm{~W}$ plasma excitation power. The strong radiation lines were identified using data from NIST [8].

\section{Conclusion and Future Work}

The factors related to electron beam power were identified. An important factor is the pressure in the plasma chamber, which was measured and it is within 0.9 to 1.2 mbar. A separate set up was put together to focus on the study of the plasma generated. The strong radiation lines in the recorded optical spectra of the argon plasma generated were identified using the NIST database. The results showed that the emission spectrum of the plasma is dependent of the plasma chamber pressure. The ratio of argon lines 750.4 (ground state) to 480.6 (single ionized) increases with the plasma chamber pressure. This could be used as an indication of increasing ionization with plasma chamber pressure. The present beam power achievable limits applications to those at lower power such as additive manufacturing and surface modification. Future work will use the optical emission spectroscopy system developed for this study to investigate the properties of other gases (i.e. krypton and neon) allowing a more detailed understanding of the effects of various parameters on the level of beam current produced. Design modifications to the plasma chamber will be carried out in order to be able to look at the plasma through the plasma chamber walls while an electron beam is being generated from it.

\section{Acknowledgements}

The authors wish to acknowledge funding from TWI and Brunel University for carrying out this work.

\section{References}

1. del Pozo, S., C. N. Ribton, and D. R. Smith, “A novel $\mathrm{RF}$ excited plasma cathode electron beam gun design”, IEEE Transactions on Electron Devices, DOI 10.1109/TED.2014.2299339.

2. del Pozo, S., C. N. Ribton, and D. R. Smith, "Spectroscopic measurement in a novel RF excited plasma cathode electron beam gun design”, accepted for presentation at IVEC'14, Monterey, CA, 2014.

3. N. Rempe et al., Welding and Cutting, 11(2), 2012.

4. A. Sanderson, "Electron beam welding generators", U.S. Patent 4072356, Feb. 7, 1978.

5. C.N. Ribton et al., "Plasma source apparatus and method for generating charged particle beams", WIPO Patent application WO 2013/186523 A1, Dec. 19, 2013.

6. U. Fantz, "Basics of plasma spectroscopy", Plasma Sources Sci. Technol. 15, pp. S137-S147, Oct. 2006.

7. Ocean Optics Inc. Optical Fiber Assemblies, [Online]. Available: www.OceanOptics.com.

8. Kramida, A., Y. Ralchenko, and J. Reader, and NIST ASD Team, 2013. NIST Atomic Spectra Database (ver. 5.1), [Online]. Available: http://physics.nist.gov/asd. 